\title{
以氨气为氢源的电化学烯丙位氢化脱氟反应研究
}

\author{
盛 杰 ${ }^{a}$ \\ 吴 娜 ${ }^{a}$ \\ 刘旭 ${ }^{a}$ \\ 刘 峰 ${ }^{a}$ \\ 刘帅 ${ }^{a}$ \\ 丁伟杰 ${ }^{a}$ \\ 刘 畅 ${ }^{a}$ \\ 程旭*, $a, b$
}

( ${ }^{a}$ 南京大学化学化工学院＼cjkstart江苏省先进有机材料重点实验室＼cjkstart化学与生物医学科学研究所＼cjkstart南京 210023)

( ${ }^{b}$ 南开大学 元素有机化学国家重点实验室 天津 30071)

\begin{abstract}
摘要 偕位二氟烯烃在药物设计及含氟分子构建等方面都有广泛的应用. 其电化学合成研究的开展有限, 主要集中于 利用三氟甲酮的脱氟烯醇硅醚化反应. 利用 $\beta$-三氟甲基肉桂酸酯的烯丙位氢化脱氟制备偕二氟烯烃的方法简单直接, 但相关报道有限. 利用电化学方法, 以氨气为氢源, 以石墨毡为阴阳极材料, 实现了一系类的 $\beta$-三氟甲基肉桂酸酯电化 学烯丙位氢化脱氟反应，以中等到优秀的收率获得了多种 $\alpha$-取代偕二氟苯乙烯类化合物. 反应中氨气与石墨䩞阴极的 组合可以高效地抑制阴极析氢的反应, 确保阴极向底物的电子转移的高选择性. 循环伏安和方波伏安实验表明反应是 经历分步的电子转移过程, 从而实现 $\mathrm{C}-\mathrm{H}$ 键的形成以及 $\mathrm{C}-\mathrm{F}$ 的断裂.
\end{abstract}

关键词 电化学; 氨气; $\mathrm{C}-\mathrm{F}$ 键活化; 石墨段电极

\section{Electrochemical Allylic Hydrodefluorination Reaction Using Gaseous Ammonia as Hydrogen Source}

\author{
Sheng, Jie ${ }^{a} \quad \mathrm{Wu}, \mathrm{Na}^{a} \quad \mathrm{Liu} \mathrm{Xu}^{a} \quad$ Liu, Feng $^{a} \quad$ Liu, Shuai $^{a}$ \\ Ding, Weijie ${ }^{a} \quad$ Liu, Chang ${ }^{a} \quad$ Cheng, $\mathrm{Xu}^{*, a, b}$ \\ ( ${ }^{a}$ Institute of Chemical and Biomedical Science, Jiangsu Advanced Organic Material Laboratory, School of Chemistry and \\ Chemical Engineering, Nanjing University, Nanjing 210023) \\ ( ${ }^{b}$ State Key Laboratory of Elemento-organic Chemistry, Nankai University, Tianjin 300071)
}

\begin{abstract}
Difluoroalkenes have wide applications in the drug designs and act as the synthon of molecules containing fluoride. The current researches on the electrochemical syntheses of gem-difluoroalkenes are limited to the silylation of enolated trifluoromethyl ketones. Herein, by using graphite felt as electrodes, the electrochemical allylic hydrodefluorination of $\alpha$-trifluoromethyl cinnamates is realized using gaseous ammonia as hydrogen source, giving gem-difluorostyrenes in moderate to good yields. The usage of ammonia and graphite felt cathode is important to inhibit the cathodic hydrogen evolution, keeping the electron transfer from cathode to substrate with high selectivity. The cyclic voltammetry $(\mathrm{CV})$ and square wave voltammetry (SWV) analyses support a stepwise electron transfer process to achieve the $\mathrm{C}-\mathrm{H}$ bond formation and $\mathrm{C}-\mathrm{F}$ bond cleavage.
\end{abstract}

Keywords electrochemistry; ammonia; $\mathrm{C}-\mathrm{F}$ bond activation; graphite felt electrode

\section{Introduction}

gem-Difluoroalkene was an important fluoro synthon in a variety of transformations and applications (Scheme 1, a). For example, $\alpha$-aminometheneyl gem-difluorostyrene was the inhibitor of monoamine oxidase (MAO) ${ }^{[1]}$ and pesticide building block. ${ }^{[2]}$ gem-Difluoroalkenes are reactive sub- strate in a variety of transformation to build complicated molecules. ${ }^{[3]}$ For example, Hu et al. ${ }^{[4]}$ achieved the AgFmediated fluorinative cross-coupling of gem-difluoroalkenes. Feng and Loh et al. ${ }^{[5]}$ utilized the gem-difluoroalkenes as starting material in a series of transitionalmetal-catalyzed and photoredox catalysis transformations.

* Corresponding author. E-mail: chengxu@nju.edu.cn

Received June 29, 2020; revised July 23, 2020; published online August 5, 2020

Dedicated to the 40th anniversary of Chinese Journal of Organic Chemistry.

Project supported by the National Natural Science Foundation of China (Nos. 22071105, 22031008), the QingLan Project of Jiangsu Education Department and the National Key Research and Development Program of China (No. 2019YFC0408303).

国家自然科学基金(No. 22071105, 22031008)、江苏省教育厅青蓝工程和国家重点研发计划(No. 2019YFC0408303)资助项目. 
(a) Applications of gem-difluoroalkenes<smiles>NCC(=C(F)F)c1ccccc1</smiles>

MAO inhibitor<smiles>CCC(=CF)CC(=O)O</smiles>

pesticide building block

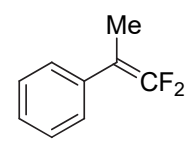

$\mathrm{CF}_{3}$ precuso (c) This work: allylic hydrodefluorination

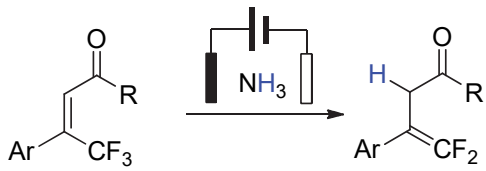

(b) Reported syntheses

Matsuda, Kitazume

Liu et al.<smiles>C#CCOC(=O)C(F)(F)Br</smiles><smiles>[R]C(=CC(=O)OCC)C(F)(F)Br</smiles><smiles>[CH]1CCC1</smiles><smiles>[R]C(CC(=O)OCC)=C(F)F</smiles><smiles>[B]C#CCOC(=O)C(F)(F)Br</smiles><smiles>CCOC(O)C(F)(F)S(=O)(=[In])OC</smiles><smiles>CCCCCCO[GeH3]</smiles><smiles>[R]C([R])=C(F)F</smiles>

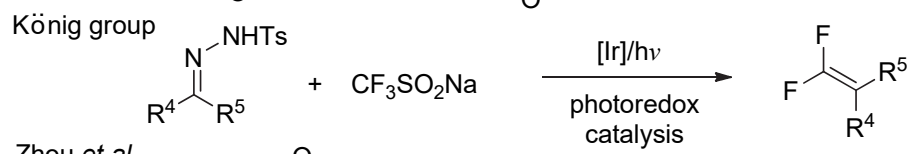
Zhou et al.<smiles>CCOC(=O)C=C(c1ccccc1)C(F)(F)F</smiles>

EtOOC<smiles></smiles>

Scheme 1 Applications and syntheses of gem-difluoroalkenes

The gem-difluorostyrenes were also applied in the thiolation by Dilman group ${ }^{[6]}$ and fluorohalogenation by Jiang group. ${ }^{[7]}$ With these divergent applications, the syntheses of gem-difluoroalkenes were extensively studied using a variety of protocols. ${ }^{[8]}$ For example, by using bromodifluoroacetate as starting material, the gem-difluoroalkene ester was prepared in 4 steps by Matsuda and Kitazume (Scheme 1b). ${ }^{[9]}$ With the same starting material, the preparation was realized via Julia-Kochienski olefination in 3 steps by the Liu group (Scheme 1b). ${ }^{[10]}$ König group ${ }^{[11]}$ reported an photoredox cross-coupling reaction between ketone-derived hydrazone and sodium trifluoromethanesulfonate catalyzed by Ir complex (Scheme 1b). Xiao et $a l^{[12]}$ achieved the $\mathrm{ZnO}$-promoted Wittig reaction. There has been one substrate reported by Zhou et al. ${ }^{[13]}$ as an unexpected reaction in the Ni-catalyzed transfer hydrogenation reaction in which the gem-difluoror styrene $\mathbf{2 a}$ was obtained (Scheme 1b). Despite no further investigation, this example suggested a potential allylic-hydrodefluorination protocol to prepare the gem-difluoroalkenes.

Electrochemical synthesis recently emerged as a powerful strategy to prepare molecules with innovative pathways. ${ }^{[14]}$ The electrochemical dechlorination and debromination reactions have been well-established. ${ }^{[15]}$ However, the direct cleavage of $\mathrm{C}-\mathrm{F}$ remains a challenging task due to strong bond energy. For example, activation of the $\mathrm{Ar}-\mathrm{F}$ requires the synergistic catalysis involving photocatalyst and electrolysis in an oxidation process. ${ }^{[16]}$ So far, the cathodic reduction of $\mathrm{C}-\mathrm{F}$ bonds focused on the conversion of trifluoromethyl ketones to silyl gem-difluoroenol ether. ${ }^{[17]}$ We reasoned that the cathodic reductive hydrodefluorination is hard to achieve due to the choice of hydrogen source. As the $\mathrm{C}-\mathrm{F}$ activation on cathode requires the high reduction potential, the cathodic hydrogen evolution might be predominant at such conditions. Recently, we ${ }^{[18]}$ reported the electrochemical hydrogenation reaction using gaseous ammonia as the hydrogen source. With the combination of graphite felt cathode and ammonia, the cathodic hydrogen evolution could be inhibited at $-2.0 \mathrm{~V}$ (vs. SCE). Herein, we reported the electrochemi- cal allylic hydrodefluorination reaction with gaseous ammonia as hydrogen source.

\section{Results and discussion}

At onset of the study, compound 1a was used as the model substrate to screen various parameters of the allylic hydrodefluorination reaction (Table 1). With graphite felt as both anode and cathode, using gaseous $\mathrm{NH}_{3}$ at balloon pressure, the desired transformation took place readily at room temperature in $\mathrm{MeCN}$ with ${ }^{n} \mathrm{Bu}_{4} \mathrm{NBF}_{4}$ as supporting electrolyte, giving product $2 \mathrm{a}$ in $78 \%$ NMR yield and $66 \%$ isolated yield (Entry 1). As expected, in the absence either electricity (Entry 2) or gaseous ammonia (Entry 3), no conversion was observed. Other supporting electrolytes instead of ${ }^{n} \mathrm{Bu}_{4} \mathrm{NBF}_{4}$ were evaluated, and the NMR yield were inferior to that obtained in standard conditions (Entries $4 \sim 7$ ). When the reaction was carried out in other solvents than $\mathrm{MeCN}$, for example dimethyl sulfoxide (DMSO) and $\mathrm{MeOH}$, the yields dropped dramatically. On the other hand, $N, N$-dimethylformamide (DMF) gave almost the identical yield to MeCN (Entry 10). Next, the constant current method was applied instead of controlled cell potential, and found constant current at a density of 15 $\mathrm{mA} / \mathrm{cm}^{3}$ could offer $66 \%$ yield (Entry 11 ). It was noted as the graphite felt was porous material, the planar surface did not make sense. The volume of graphite felt was used in the expression of current density. Varied cell potentials were examined as well, the yield increased from $49 \%$ to $78 \%$ when the cell potential moved up from 2.8 to $3.2 \mathrm{~V}$. Further elevation of cell potential resulted in the decrease of yields (Entry 12). This observation was in accord with the results from constant current experiments in Entry 11. Finally, the cathode material was comparaed. By changing the graphite felt to graphite rod or platinum, the reaction was inhibited (Entries 13, 14).

With the optimal conditions in hand (Table 1, Entry 1), the substrate scope of electrochemical hydrodefluorination reaction was explored (Table 2 ). The methyl substitution in substrates $\mathbf{1 b}$ and 1c unexpectedly sabotaged the reaction 
Table 1 Reaction optimization of electrochemical hydrodefluorination reaction ${ }^{a}$<smiles>CCOC(=O)/C=C(\C(=O)OCC)c1ccccc1</smiles>

\begin{tabular}{clc}
\hline Entry & \multicolumn{1}{c}{ Variation from standard condition } & Yield ${ }^{b} \%$ \\
\hline 1 & None & $78\left(66^{c}\right)$ \\
2 & No voltage & 0 \\
3 & $\mathrm{No} \mathrm{NH}_{3}$ & 0 \\
4 & $\mathrm{LiClO}_{4}$ instead of ${ }^{n} \mathrm{Bu}_{4} \mathrm{NBF}_{4}$ & 62 \\
5 & $\mathrm{LiBF}_{4}$ instead of ${ }^{n} \mathrm{Bu}_{4} \mathrm{NBF}_{4}$ & 54 \\
6 & $n \mathrm{Bu}_{4} \mathrm{NPF}_{6}$ instead of ${ }^{n} \mathrm{Bu}_{4} \mathrm{NBF}_{4}$ & 67 \\
7 & $n \mathrm{Bu}_{4} \mathrm{NClO}_{4}$ instead of ${ }^{n} \mathrm{Bu}_{4} \mathrm{NBF}_{4}$ & 71 \\
8 & $\mathrm{DMSO}$ as solvent & 48 \\
9 & $\mathrm{MeOH}$ as solvent $_{1}$ & 32 \\
10 & DMF as solvent & 76 \\
11 & $\mathrm{Constant}$ current is $55,111,167,222 \mathrm{~mA} / \mathrm{g}^{d}$ & $23,47,66,42$ \\
12 & Constant voltage is $2.8,3.0,3.4,3.6 \mathrm{~V}$ & $49,59,68,28$ \\
13 & Graphite rod instead of graphite felt & 14 \\
\hline
\end{tabular}

${ }^{a}$ Standard condition: 1a $(0.2 \mathrm{mmol}),{ }^{n} \mathrm{Bu}_{4} \mathrm{NBF}_{4}\left(0.3\right.$ equiv.), $\mathrm{CH}_{3} \mathrm{CN}(5 \mathrm{~mL})$, graphite felt anode and cathode, undivided cell, $3.2 \mathrm{~V}$ cell voltage, r.t., $\mathrm{NH}_{3}$ atmosphere, 5 h. ${ }^{b}$ Yields determined by ${ }^{1} \mathrm{H}$ NMR with dichloromethane as the internal standard. ${ }^{c}$ Isolated yield. ${ }^{d}$ As graphite felt is the complex of fibre, the $\mathrm{mA} / \mathrm{g}$ is more suitable to describe the constant current condition.

Table 2 Scope of substrates ${ }^{a}$
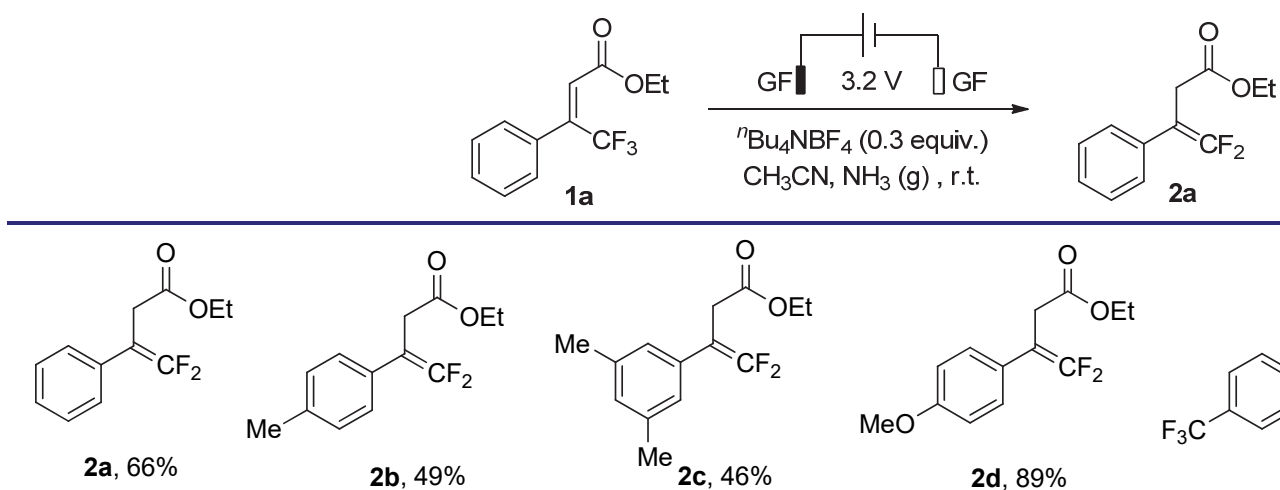<smiles>C=C=C(CC(=O)OCC)c1ccc(C(F)(F)F)cc1</smiles>

2e, $59 \%$<smiles>C=Cc1ccc(C(=C=[Pt])CC(=O)OCC)cc1</smiles>

$2 \mathrm{~g}, 70 \%$<smiles>CCOC(=O)CC(=C(F)F)c1ccc(Br)cc1</smiles>

2m, $66 \%$<smiles>CCCCOC(=O)CC(=C(F)F)c1ccccc1</smiles>

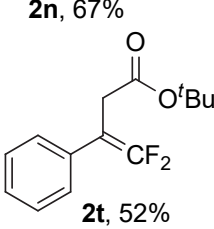<smiles>CCOC(=O)CC(=C(F)F)c1ccc(F)cc1</smiles>

2i, $62 \%$<smiles>CCOC(=O)CC(=C(F)F)c1cccc(F)c1</smiles>

2j, $62 \%$<smiles>CCOC(=O)CC(=C=[Fe])c1ccc(C(C)(C)C)cc1</smiles>

2f, $44 \%$<smiles>CCOC(=O)CC(=C(F)F)c1ccc(Cl)cc1</smiles>

2k, $52 \%$<smiles>CCOC(=O)CC(=C=[Fe])c1cccc(Cl)c1</smiles>

2I, $41 \%$<smiles>CCOC(=O)CC(=C=[P+])c1coc2ccccc12</smiles>

2o, $78 \%$<smiles>CCOC(=O)CC(=C(F)F)c1cc2ccccc2c2ccccc12</smiles><smiles>NC(=O)CC(=C(F)C(F)(F)F)c1cccc2ccccc12</smiles>

2q, $90 \%$<smiles>CCOC(=O)CC(=C(F)c1ccsc1)c1ccsc1</smiles><smiles>C=CCOC(=O)CC(=C(F)F)c1ccccc1</smiles><smiles>O=C(CC(=C(F)c1ccccc1)C(F)(F)F)OCC(F)(F)F</smiles><smiles>O=C(CC(=C(F)F)c1ccccc1)NCc1ccccc1</smiles>

${ }^{a}$ Conditions: $1(0.2 \mathrm{mmol}),{ }^{n} \mathrm{Bu}_{4} \mathrm{NBF}_{4}\left(0.3\right.$ equiv.), $\mathrm{CH}_{3} \mathrm{CN}(5 \mathrm{~mL})$, graphite felt anode and cathode, undivided cell, $3.2 \mathrm{~V}$ cell potential, r.t., $\mathrm{NH}_{3}$ balloon, $5 \mathrm{~h}$, isolated yield. 
yield substantially, due to overdefluorination reaction. It is gratifying to isolate the product $\mathbf{2 d}$ with $p$-methoxy substitution in $89 \%$ yield. Electron-withdrawing group, for example, $\mathrm{CF}_{3}$ group, impact the yield with a $59 \%$ yield. The product $2 \mathbf{f}$ with para ${ }^{t} \mathrm{Bu}$ group was obtained in moderate yield. It was glad to observe that the vinyl group in product $2 \mathrm{~g}$ was intact and the corresponding yield was $70 \%$. The good yield of $\mathbf{2 h}$ was also realized in $5 \mathrm{~h}$. Next, a series of substrates $\mathbf{1 i} \sim \mathbf{1 n}$ bearing halogen atom were subject to the standard hydrodefluorination reaction. The yields were around $60 \%$, except the products $2 \mathbf{k}$ and $2 \mathbf{l}$ containing $\mathrm{Cl}$ atom. The low yields of both products were majorly caused by the extensive purification. Especially, the para-bromo atom in product $\mathbf{2 m}$ survived the electrochemical process, demonstrating the functional group compatibility of this protocol. Products $\mathbf{2 0} \sim \mathbf{2 q}$ bearing fused rings were achieved in good yields. Gladly, product $2 \mathbf{r}$ containing thiophenyl group could be prepared in $84 \%$ isolated yield. Subsequently, the substrates other than ethyl ester were prepared and evaluated. Several esters $1 \mathbf{s} \sim \mathbf{1} \mathbf{u}$, bearing $n$-butyl, $t$-butyl or allyl group, underwent the transformation readily, and the desired products $\mathbf{2 s} \sim \mathbf{2 u}$ were generated in yields from $52 \%$ to $87 \%$. When trifluoroethyl ester 1v was employed as starting material, the desired product $2 \mathbf{v}$ was obtained in only $42 \%$ yield partially due to the in situ transamidation reaction. Finally, by using an amide as starting material, product $\mathbf{2} \mathbf{w}$ was obtained in good yield $(81 \%)$.

Next, the electrochemistry analyses were conducted to gain further information of this transformation. The cyclic voltammetry experiments were performed to study the cathodic reduction process (Figure 1, a). At first, the ammonia solution showed totally inert during the whole scan. By deducting the signals of blank sample, the first reduction peak of 1a was observed at $-2.0 \mathrm{~V}$ (vs. SCE). When the cyclic voltammetry $(\mathrm{CV})$ analysis was carried out under gaseous ammonia, a second weak peak was detected at $-2.2 \mathrm{~V}$ (vs. SCE). In order to separate these two peaks, square wave voltammetry (SWV) experiment was carried out (Figure 1, b). With the significant separation at a frequency of $10 \mathrm{~Hz}$, a two-step electron transfer was supported.

With the data achieved, a plausible reaction pathway was proposed in Scheme 2. At first the cathodic electron transfer converts substrate, for example 1a, to its' corresponding radical anion $\mathbf{A}$, in which $\mathbf{C}(2)$ was supposed to hold more negative charge because of the carbonyl group. The protonation takes place at this position at first with ammonium cation generated via anodic oxidation of ammonia. A neutral radical $\mathbf{B}$ was produced. Consequently, a second anodic electron transfer gives rise to the benzylic anion $\mathbf{C}$ that undergoes an elimination to desired product 2 a.

\section{Conclusions}

In summary, an electrochemical hydrodefluorination
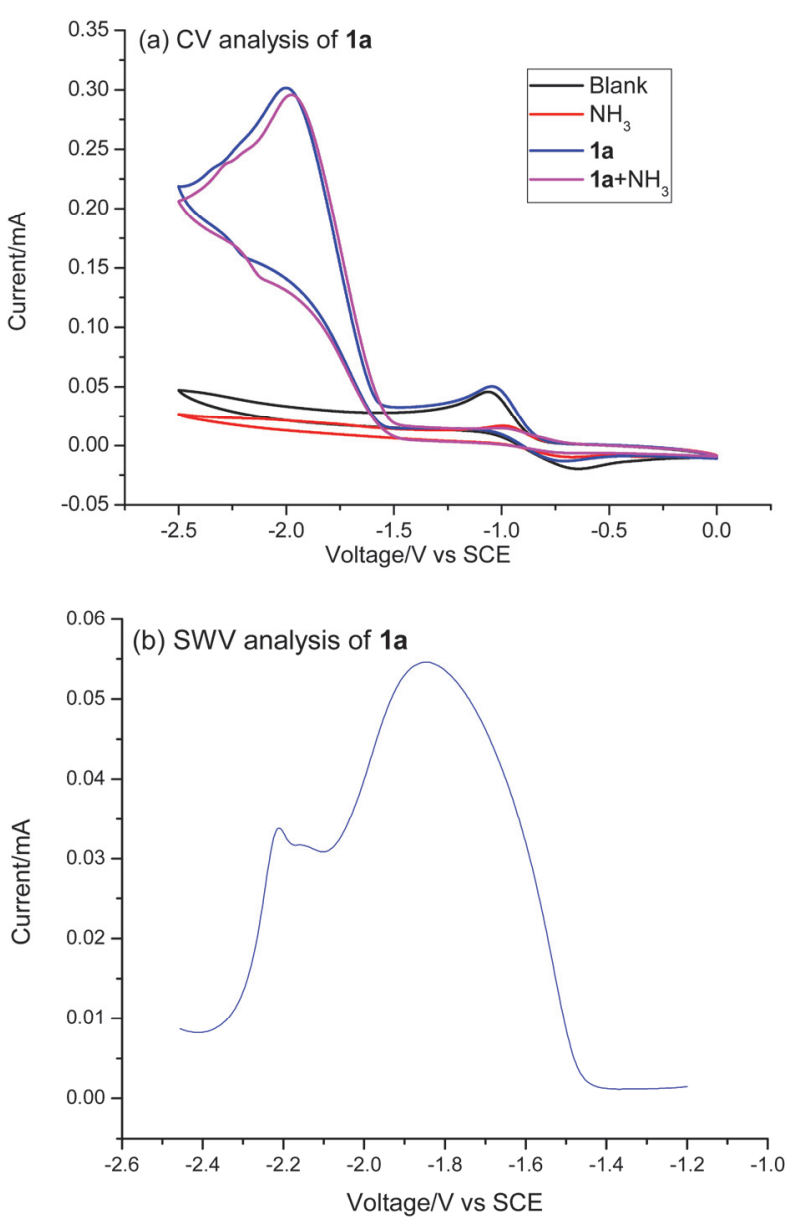

Figure 1 Electrochemistry analyses of $\mathbf{1 a}$ (a) CV condition: glassy carbon working electrode, $\mathrm{Pt}$ wire counter electrode, SCE reference electrode, $50 \mathrm{mV} / \mathrm{S}$. (b) 1 a $(0.04$ $\mathrm{mmol}),{ }^{n} \mathrm{Bu}_{4} \mathrm{BF}_{4}(0.012 \mathrm{~mol} / \mathrm{L}), \mathrm{MeCN}(5 \mathrm{~mL})$, pulse height 25 $\mathrm{mV}$, step height $4 \mathrm{mV}$, frequency $10 \mathrm{~Hz}$

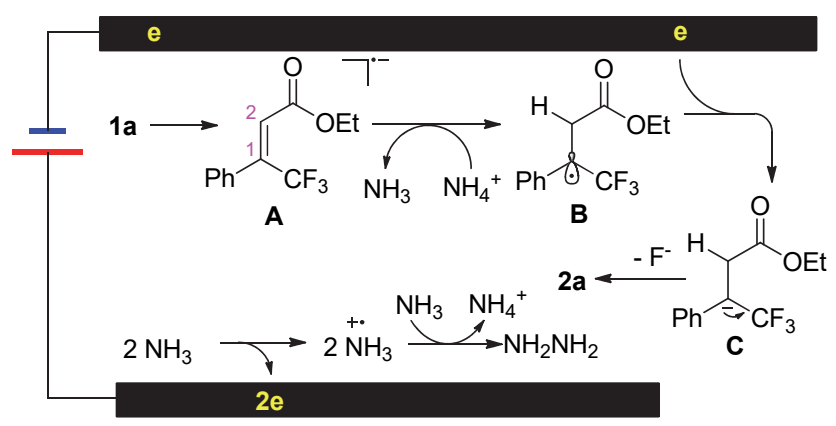

Scheme 2 Plausible reaction pathway of electrochemical defluorinative hydrogenation

reaction with gem-difiluoroalkene esters as products was developed. The catalyst-free reaction employs inexpensive graphite felt as both anode and cathode, ammonia as a safe and environment-friendly hydrogen source. With this protocol, a series of $\alpha$-trifluoromethyl cinnmates were converted to the gem-difluoro styrenes in moderate to good yields. The CV and SWV analyses supported a two-step electron transfer pathway. Especially, the reaction showed that the combination of ammonia and graphite felt cathode 
was a highly efficient approach to perform cathodic reduction with substrates requiring high reduction potential.

\section{Experimental section}

\subsection{Instruments and reagents}

Column chromatography was generally performed on silica gel $(300 \sim 400$ mesh) and reactions were monitored by thin-layer chromatography (TLC) using $254 \mathrm{~nm} \mathrm{UV}$ light to visualize the course of the reactions. NMR spectra were recorded on a Bruker Avance $400 \mathrm{MHz}$ spectrometer. High resolution mass spectra were recorded on Agilent G6500 Q-TOF instrument. Electrochemical analyses were carried out on $\mathrm{CHI} 730 \mathrm{E}$ electrochemistry workstation. ${ }^{1} \mathrm{H}$ NMR (400 MHz), ${ }^{13} \mathrm{C}$ NMR (101 MHz) and ${ }^{19} \mathrm{~F}(376 \mathrm{MHz})$ were recorded on Bruker Avance III $400(400 \mathrm{MHz}$ and 100 $\mathrm{MHz}$ ). All ${ }^{1} \mathrm{H} \mathrm{NMR}$ and ${ }^{13} \mathrm{C}$ NMR spectra are reported in parts per million (ppm) downfield of TMS. High-resolution mass spectra (HRMS) were equipped with an ESI source and a TOF detector mass spectrometer. The melting points were measured with digital melting point detector.

All reactions that required anhydrous conditions were carried by standard procedures under nitrogen atmosphere. Unless otherwise noted, materials (AR grade) were purchased from commercial suppliers, such as J\&K, Innochem, and Energy chemicals, and used without further purification. The solvents were dried by distillation over the appropriate drying reagents.

\subsection{General procedure of the electrochemical allylic hydrodefluorination reaction}

A $10 \mathrm{~mL}$ two-necked heart-shaped flask was charged with the substrate $1 \mathrm{a}(0.2 \mathrm{mmol}, 1.0$ equiv. $),{ }^{n} \mathrm{Bu}_{4} \mathrm{NBF}_{4}$ ( 0.06 mmol, 0.3 equiv.) and a magnetic stir bar. The flask was equipped with a rubber stopper. And graphite felt (2 $\mathrm{cm} \times 1 \mathrm{~cm} \times 0.5 \mathrm{~cm}$ ) was used as anode and cathode. Two electrodes were separated with a Teflon film. The graphite felt anode was attached to a platinum wire and cathode was attached to a silver wire. A Teflon wire was tied around two electrodes. The flask was evacuated and backfilled with $\mathrm{NH}_{3}$, and then $\mathrm{CH}_{3} \mathrm{CN}(5 \mathrm{~mL})$ was added by syringe. The mixture was stirred under room temperature and controlled cell potential electrolysis. After the reaction was completed by monitoring with TLC or GC-MS analysis, the mixture was diluted with water and extracted with EtOAc twice. The combined organic layers were washed with brine, dried over $\mathrm{Na}_{2} \mathrm{SO}_{4}$, filtered and concentrated. The residue was purified by chromatography on silica gel to afford the desire product $\mathbf{2 a}$. Copounds $\mathbf{2} \mathbf{b} \sim \mathbf{2} \mathbf{w}$ were synthesized using the same method.

Ethyl 4,4-difluoro-3-phenylbut-3-enoate (2a): $29 \mathrm{mg}$, yield $66 \%$, chromatography on silica gel [ $V$ (petroleum ether $): V($ EtOAc $)=98: 2]$, colorless oil. ${ }^{1} \mathrm{H}$ NMR $(400$ MHz, Chloroform- $d$ ) $\delta$ : $7.35(\mathrm{~d}, J=6.1 \mathrm{~Hz}, 4 \mathrm{H}), 7.31 \sim$ 7.27 (m, 1H), 4.12 (q, $J=7.1 \mathrm{~Hz}, 2 \mathrm{H}), 3.40$ (t, $J=2.2 \mathrm{~Hz}$, 2H), $1.20(\mathrm{t}, J=7.1 \mathrm{~Hz}, 3 \mathrm{H}) ;{ }^{13} \mathrm{C} \mathrm{NMR}\left(101 \mathrm{MHz}, \mathrm{CDCl}_{3}\right)$ $\delta: 170.20 \sim 170.10(\mathrm{~m}), 154.79(\mathrm{dd}, J=292.3,289.0 \mathrm{~Hz})$, $133.03(\mathrm{t}, J=3.8 \mathrm{~Hz}), 128.49,127.84(\mathrm{t}, J=3.5 \mathrm{~Hz}), 87.16$ (dd, $J=21.6,17.9 \mathrm{~Hz}), 127.53,61.10,33.89$ (d, $J=2.6$ $\mathrm{Hz}), \quad 14.05 ;{ }^{19} \mathrm{~F}$ NMR $(376 \mathrm{MHz}$, Chloroform- $d) \quad \delta$ : $-86.38(\mathrm{~d}, J=34.8 \mathrm{~Hz}),-89.22$ (d, $J=34.8 \mathrm{~Hz})$; HRMS (ESI) calcd for $\mathrm{C}_{12} \mathrm{H}_{13} \mathrm{~F}_{2} \mathrm{O}_{2}[\mathrm{M}+\mathrm{H}]^{+}$227.0803, found 227.0873 .

Ethyl 4,4-difluoro-3-(p-tolyl)but-3-enoate (2b): $24 \mathrm{mg}$, yield $49 \%$, chromatography on silica gel [ $V$ (petroleum ether $): V($ EtOAc $)=98: 2]$, colorless oil. ${ }^{1} \mathrm{H}$ NMR $(400$ $\mathrm{MHz}$, Chloroform- $d$ ) $\delta: 7.23$ (d, $J=7.9 \mathrm{~Hz}, 2 \mathrm{H}), 7.16$ (d, $J=8.0 \mathrm{~Hz}, 2 \mathrm{H}), 4.12$ (q, $J=7.2 \mathrm{~Hz}, 2 \mathrm{H}), 3.38$ (s, 2H), 2.34 $(\mathrm{s}, 3 \mathrm{H}), 1.20(\mathrm{t}, J=7.1 \mathrm{~Hz}, 3 \mathrm{H}) ;{ }^{13} \mathrm{C} \mathrm{NMR}(101 \mathrm{MHz}$, Chloroform- $d$ ) $\delta$ : 170.19 (dd, $J=4.2,2.9 \mathrm{~Hz}$ ), 154.72 (dd, $J=291.8,288.7 \mathrm{~Hz}), 137.32,130.03(\mathrm{t}, J=3.8 \mathrm{~Hz})$, $129.19,127.70(\mathrm{t}, J=3.5 \mathrm{~Hz}), 87.01$ (dd, $J=21.3,18.0$ $\mathrm{Hz}), 61.03,33.92(\mathrm{~d}, J=2.6 \mathrm{~Hz}), 21.09,14.06 ;{ }^{19} \mathrm{~F}$ NMR (376 MHz, Methanol- $d_{4}$ ) $\delta$ : -91.09 (d, $J=40.1 \mathrm{~Hz}$ ), - $92.25(\mathrm{~d}, J=39.6 \mathrm{~Hz})$; HRMS (ESI) calcd for $\mathrm{C}_{13} \mathrm{H}_{15} \mathrm{~F}_{2} \mathrm{O}_{2}[\mathrm{M}+\mathrm{H}]^{+}$241.1040, found 241.1033.

Ethyl 3-(3,5-dimethylphenyl)-4,4-difluorobut-3-enoate (2c): $23 \mathrm{mg}$, yield: 46\%, chromatography on silica gel $[V($ petroleum ether $): V($ EtOAc $)=98: 2]$, colorless oil. ${ }^{1} \mathrm{H}$ NMR (400 MHz, Chloroform- $d$ ) $\delta: 6.95$ (s, 2H), 6.92 (s, $1 \mathrm{H}), 4.13$ (q, $J=7.1 \mathrm{~Hz}, 2 \mathrm{H}), 3.36(\mathrm{~s}, 2 \mathrm{H}), 2.31$ (s, 6H), $1.21(\mathrm{t}, J=7.1 \mathrm{~Hz}, 3 \mathrm{H}) ;{ }^{13} \mathrm{C}$ NMR $(101 \mathrm{MHz}$, Chloroform- $d$ ) $\delta: 170.22(\mathrm{dd}, J=4.2,2.9 \mathrm{~Hz}), 154.77$ (dd, $J=$ 291.9, 288.7 Hz), 137.96, 132.91 (t, $J=3.8 \mathrm{~Hz}$ ), 130.14, $129.27,126.67,125.68$ (t, $J=3.4 \mathrm{~Hz}), 87.19$ (dd, $J=21.0$, $18.1 \mathrm{~Hz}), 61.01,34.02(\mathrm{~d}, J=2.7 \mathrm{~Hz}), 21.30,14.08 ;{ }^{19} \mathrm{~F}$ NMR (376 MHz, Methanol-d $\left.d_{4}\right) \delta$ : -90.46 (d, $J=39.1$ $\mathrm{Hz}$ ), $-91.46(\mathrm{~d}, J=39.1 \mathrm{~Hz}$ ); HRMS (ESI) calcd for $\mathrm{C}_{14} \mathrm{H}_{17} \mathrm{~F}_{2} \mathrm{O}_{2}[\mathrm{M}+\mathrm{H}]^{+}$255.1196, found 255.1187.

Ethyl 4,4-difluoro-3-(4-methoxyphenyl)but-3-enoate (2d): $46 \mathrm{mg}$, yield: $89 \%$, chromatography on silica gel $[V($ petroleum ether $): V($ EtOAc $)=98: 2]$, colorless oil. ${ }^{1} \mathrm{H}$ NMR (400 MHz, Chloroform-d) $\delta: 7.26(\mathrm{~d}, J=8.6 \mathrm{~Hz}$, $2 \mathrm{H}), 6.88(\mathrm{~d}, J=8.4 \mathrm{~Hz}, 2 \mathrm{H}), 4.12(\mathrm{q}, J=7.1 \mathrm{~Hz}, 2 \mathrm{H}), 3.80$ $(\mathrm{s}, 3 \mathrm{H}), 3.36(\mathrm{~s}, 2 \mathrm{H}), 1.20(\mathrm{t}, J=7.1 \mathrm{~Hz}, 3 \mathrm{H}) ;{ }^{13} \mathrm{C} \mathrm{NMR}$ $\left(101 \mathrm{MHz}, \mathrm{CDCl}_{3}\right) \delta: 170.22(\mathrm{dd}, J=4.2,2.9 \mathrm{~Hz}), 158.89$, $154.63(\mathrm{dd}, J=291.0,288.5 \mathrm{~Hz}), 129.05$ (t, $J=3.5 \mathrm{~Hz})$, $125.21(\mathrm{t}, J=3.8 \mathrm{~Hz}), 113.96,86.69(\mathrm{dd}, J=21.6,18.1$ $\mathrm{Hz}), 61.03,55.22,34.03(\mathrm{~d}, J=2.6 \mathrm{~Hz}), 14.07 ;{ }^{19} \mathrm{~F}$ NMR (376 MHz, Methanol- $\left.d_{4}\right) \delta$ : $-91.74(\mathrm{~d}, J=41.5 \mathrm{~Hz}$ ), - $92.87(\mathrm{~d}, J=41.9 \mathrm{~Hz})$; HRMS (ESI) calcd for $\mathrm{C}_{13} \mathrm{H}_{15} \mathrm{~F}_{2} \mathrm{O}_{3}[\mathrm{M}+\mathrm{H}]^{+}$257.0909, found 257.0981.

Ethyl 4,4-difluoro-3-(4-(trifluoromethyl)phenyl)but3-enoate (2e): $35 \mathrm{mg}$, yield 59\%, chromatography on silica gel $[V$ (petroleum ether) $: V($ EtOAc $)=98: 2]$, colorless oil. ${ }^{1} \mathrm{H}$ NMR (400 MHz, Chloroform-d) $\delta: 7.61$ (d, $J=8.1$ $\mathrm{Hz}, 2 \mathrm{H}), 7.47$ (d, $J=8.1 \mathrm{~Hz}, 2 \mathrm{H}), 4.13$ (q, $J=7.1 \mathrm{~Hz}, 2 \mathrm{H})$, 3.42 (s, 2H), 1.20 (t, $J=6.9 \mathrm{~Hz}, 3 \mathrm{H}) ;{ }^{13} \mathrm{C} \mathrm{NMR} \mathrm{(101} \mathrm{MHz,}$ Chloroform- $d$ ) $\delta$ : 169.75 (dd, $J=4.1,2.7 \mathrm{~Hz}), 155.13$ (dd, $J=294.1,290.8 \mathrm{~Hz}), 128.20$ (t, $J=3.7 \mathrm{~Hz}), 125.68$ (q, $J=$ $3.7 \mathrm{~Hz}), 125.46$ (q, $J=3.8 \mathrm{~Hz}), 123.96(\mathrm{q}, J=272.1 \mathrm{~Hz})$, 86.72 (dd, $J=22.5,17.1 \mathrm{~Hz}), 61.29,33.55$ (d, $J=2.5 \mathrm{~Hz}$ ), 14.01; ${ }^{19} \mathrm{~F}$ NMR (376 MHz, Methanol- $\left.d_{4}\right) \delta$ : -63.81 , $-88.29(\mathrm{~d}, J=34.0 \mathrm{~Hz}),-89.85(\mathrm{~d}, J=34.2 \mathrm{~Hz})$; HRMS 
(ESI) calcd for $\mathrm{C}_{13} \mathrm{H}_{12} \mathrm{~F}_{5} \mathrm{O}_{2}[\mathrm{M}+\mathrm{H}]^{+}$295.0757, found 295.0747.

Ethyl 3-(4-(tert-butyl)phenyl)-4,4-difluorobut-3-enoate (2f): $25 \mathrm{mg}$, yield $44 \%$, chromatography on silica gel $[V($ petroleum ether $): V($ EtOAc $)=98: 2]$, colorless oil. ${ }^{1} \mathrm{H}$ NMR (400 MHz, Chloroform- $d$ ) $\delta: 7.30(\mathrm{~d}, J=8.6 \mathrm{~Hz}$, $2 \mathrm{H}), 7.20(\mathrm{~d}, J=8.9 \mathrm{~Hz}, 2 \mathrm{H}), 4.06(\mathrm{q}, J=7.1 \mathrm{~Hz}, 2 \mathrm{H}), 3.30$ (s, 2H), $1.24(\mathrm{~s}, 9 \mathrm{H}), 1.13(\mathrm{t}, J=7.1 \mathrm{~Hz}, 3 \mathrm{H}) ;{ }^{13} \mathrm{C}$ NMR $\left(101 \mathrm{MHz}, \mathrm{CDCl}_{3}\right) \delta: 170.27(\mathrm{~d}, J=7.0 \mathrm{~Hz}), 154.83(\mathrm{dd}$, $J=292.3,288.8 \mathrm{~Hz}), 150.47,130.02(\mathrm{t}, J=3.9 \mathrm{~Hz}), 127.42$ (t, $J=3.6 \mathrm{~Hz}), 125.43,86.91(\mathrm{dd}, J=21.3,17.6 \mathrm{~Hz})$, $61.05,34.52,33.88,33.85,31.22,14.06 ;{ }^{19} \mathrm{~F}$ NMR (376 MHz, Chloroform- $d$ ) $\delta:-87.15(\mathrm{~d}, J=39.9 \mathrm{~Hz}),-88.31$ (d, $J=40.0 \mathrm{~Hz}$ ); HRMS (ESI) calcd for $\mathrm{C}_{16} \mathrm{H}_{21} \mathrm{~F}_{2} \mathrm{O}_{2}[\mathrm{M}+$ $\mathrm{H}]^{+}$283.1509, found 283.1502 .

Ethyl 4,4-difluoro-3-(4-vinylphenyl) but-3-enoate (2g): $36 \mathrm{mg}$, yield $70 \%$, chromatography on silica gel $[V($ petroleum ether $): V($ EtOAc $)=98: 2]$, colorless oil. ${ }^{1} \mathrm{H}$ NMR $(400 \mathrm{MHz}$, Chloroform- $d$ ) $\delta: 7.40(\mathrm{~d}, J=8.2 \mathrm{~Hz}$, $2 \mathrm{H}), 7.31(\mathrm{~d}, J=7.8 \mathrm{~Hz}, 2 \mathrm{H}), 6.70(\mathrm{dd}, J=17.6,10.9 \mathrm{~Hz}$, $1 \mathrm{H}), 5.76(\mathrm{~d}, J=17.6 \mathrm{~Hz}, 1 \mathrm{H}), 5.26(\mathrm{~d}, J=10.8 \mathrm{~Hz}, 1 \mathrm{H})$, $4.13(\mathrm{q}, J=7.1 \mathrm{~Hz}, 2 \mathrm{H}), 3.39(\mathrm{~s}, 2 \mathrm{H}), 1.21(\mathrm{t}, J=7.1 \mathrm{~Hz}$, $3 \mathrm{H}) ;{ }^{13} \mathrm{C}$ NMR (101 MHz, Chloroform- $d$ ) $\delta: 170.08$ (dd, $J=4.1,2.8 \mathrm{~Hz}), 154.85$ (dd, $J=292.9,289.4 \mathrm{~Hz}), 136.19$, 132.40 (t, $J=4.0 \mathrm{~Hz}), 127.92(\mathrm{t}, J=3.8 \mathrm{~Hz}), 126.29$, $114.26,87.04$ (dd, $J=21.7,17.5 \mathrm{~Hz}), 61.09,33.73$ (d, $J=$ $2.8 \mathrm{~Hz}), 14.09 ;{ }^{19} \mathrm{~F}$ NMR $\left(376 \mathrm{MHz}\right.$, Methanol- $\left.d_{4}\right) \delta$ : $-90.63(\mathrm{~d}, J=38.3 \mathrm{~Hz}),-91.76(\mathrm{~d}, J=38.5 \mathrm{~Hz})$; HRMS (ESI) calcd for $\mathrm{C}_{14} \mathrm{H}_{15} \mathrm{~F}_{2} \mathrm{O}_{2}[\mathrm{M}+\mathrm{H}]^{+}$253.1040, found 253.1032.

Ethyl 3-([1,1'-biphenyl]-4-yl)-4,4-difluorobut-3-enoate (2h): $49 \mathrm{mg}$, yield $81 \%$, chromatography on silica gel $[V($ petroleum ether $): V($ EtOAc $)=95: 5]$, colorless solid. ${ }^{1} \mathrm{H}$ NMR (400 MHz, Chloroform- $d$ ) $\delta: 7.59$ (d, $J=7.8 \mathrm{~Hz}$, $4 \mathrm{H}), 7.48 \sim 7.40(\mathrm{~m}, 4 \mathrm{H}), 7.36(\mathrm{t}, J=7.4 \mathrm{~Hz}, 1 \mathrm{H}), 4.15(\mathrm{q}$, $J=7.1 \mathrm{~Hz}, 2 \mathrm{H}), 3.44(\mathrm{~s}, 2 \mathrm{H}), 1.22(\mathrm{t}, J=7.1 \mathrm{~Hz}, 3 \mathrm{H}) ;{ }^{13} \mathrm{C}$ $\operatorname{NMR}\left(101 \mathrm{MHz}, \mathrm{CDCl}_{3}\right) \delta: 170.20(\mathrm{~d}, J=2.9 \mathrm{~Hz}), 140.36$ $(\mathrm{d}, J=11.7 \mathrm{~Hz}), 132.02 \sim 131.87(\mathrm{~m}), 128.79,128.15(\mathrm{t}$, $J=3.7 \mathrm{~Hz}), 127.44,127.18,127.00,86.92$ (dd, $J=21.6$, $17.3 \mathrm{~Hz}), 61.16,33.77$ (d, $J=2.5 \mathrm{~Hz}), 14.08 ;{ }^{19} \mathrm{~F}$ NMR $(376 \mathrm{MHz}$, Chloroform- $d$ ) $\delta$ : $-86.06(\mathrm{~d}, J=38.1 \mathrm{~Hz})$, $-87.32(\mathrm{~d}, J=37.5 \mathrm{~Hz}$ ); HRMS (ESI) calcd for $\mathrm{C}_{18} \mathrm{H}_{17} \mathrm{~F}_{2} \mathrm{O}_{2}[\mathrm{M}+\mathrm{H}]^{+}$303.1196, found 303.1188.

Ethyl 4,4-difluoro-3-(4-fluorophenyl) but-3-enoate (2i): $30 \mathrm{mg}$, yield $62 \%$, chromatography on silica gel $[V($ petroleum ether $): V($ EtOAc $)=98: 2]$, colorless oil. ${ }^{1} \mathrm{H}$ NMR (400 MHz, Chloroform- $d$ ) $\delta: 7.33 \sim 7.28(\mathrm{~m}, 2 \mathrm{H})$, $7.08 \sim 7.01(\mathrm{~m}, 2 \mathrm{H}), 4.12(\mathrm{q}, J=7.1 \mathrm{~Hz}, 2 \mathrm{H}), 3.36(\mathrm{t}, J=$ $2.2 \mathrm{~Hz}, 2 \mathrm{H}), 1.20(\mathrm{t}, J=7.1 \mathrm{~Hz}, 3 \mathrm{H}) ;{ }^{13} \mathrm{C}$ NMR $(101 \mathrm{MHz}$, Chloroform- $d$ ) $\delta: 169.97$ (dd, $J=4.1,2.7 \mathrm{~Hz}), 162.01$ (d, $J=247.5 \mathrm{~Hz}), 158.06 \sim 151.53(\mathrm{~m}), 129.69$ (dt, $J=7.8,3.5$ $\mathrm{Hz}), 128.96$ (q, $J=3.6 \mathrm{~Hz}), 115.60,115.38,86.48$ (dd, $J=$ $22.2,18.2 \mathrm{~Hz}), 61.13,33.98(\mathrm{~d}, J=2.6 \mathrm{~Hz}), 14.03 ;{ }^{19} \mathrm{~F}$ NMR (376 MHz, Methanol- $\left.d_{4}\right) \delta:-90.13$ (d, $J=38.6$ $\mathrm{Hz}),-91.39(\mathrm{~d}, J=38.1 \mathrm{~Hz}),-115.41$; HRMS (ESI) calcd for $\mathrm{C}_{12} \mathrm{H}_{12} \mathrm{~F}_{3} \mathrm{O}_{2}[\mathrm{M}+\mathrm{H}]^{+}$245.0789, found 245.0782.

Ethyl 4,4-difluoro-3-(3-fluorophenyl) but-3-enoate (2j):
$30 \mathrm{mg}$, yield $62 \%$, chromatography on silica gel $[V($ petroleum ether $): V($ EtOAc $)=98: 2]$, colorless oil. ${ }^{1} \mathrm{H}$ NMR $(400 \mathrm{MHz}$, Chloroform- $d$ ) $\delta: 7.32(\operatorname{td}, J=8.1,6.0$ $\mathrm{Hz}, 1 \mathrm{H}), 7.12(\mathrm{~d}, J=7.8 \mathrm{~Hz}, 1 \mathrm{H}), 7.07(\mathrm{~d}, J=8.8 \mathrm{~Hz}, 1 \mathrm{H})$, $7.03 \sim 6.93(\mathrm{~m}, 1 \mathrm{H}), 4.14(\mathrm{q}, J=7.1 \mathrm{~Hz}, 2 \mathrm{H}), 3.38(\mathrm{t}, J=$ $2.2 \mathrm{~Hz}, 2 \mathrm{H}), 1.21(\mathrm{t}, J=7.1 \mathrm{~Hz}, 3 \mathrm{H}) ;{ }^{13} \mathrm{C}$ NMR $(101 \mathrm{MHz}$, Chloroform- $d$ ) $\delta: 169.86$ (dd, $J=4.1,2.8 \mathrm{~Hz}), 162.73$ (d, $J=245.8 \mathrm{~Hz}), 154.99(\mathrm{dd}, J=293.5,289.9 \mathrm{~Hz}), 135.18$ (dd, $J=8.3,4.2 \mathrm{~Hz}), 129.99$ (d, $J=8.5 \mathrm{~Hz}), 123.48$ (q, $J=$ $3.2 \mathrm{~Hz}), 115.10$ (dd, $J=4.4,3.3 \mathrm{~Hz}), 114.87$ (dd, $J=4.4$, $3.3 \mathrm{~Hz}), 114.52(\mathrm{~d}, J=21.0 \mathrm{~Hz}), 86.98 \sim 86.36(\mathrm{~m}), 61.22$, 33.68 (d, $J=2.6 \mathrm{~Hz}), 14.05 ;{ }^{19} \mathrm{~F}$ NMR $(376 \mathrm{MHz}$, Chloroform- $d$ ) $\delta:-86.59(\mathrm{~d}, J=32.6 \mathrm{~Hz}),-87.60(\mathrm{~d}, J=32.0$ $\mathrm{Hz}$ ), -112.81; HRMS (ESI) calcd for $\mathrm{C}_{12} \mathrm{H}_{12} \mathrm{~F}_{3} \mathrm{O}_{2}[\mathrm{M}+$ $\mathrm{H}]^{+}$245.0789, found 245.0792.

Ethyl 3-(4-chlorophenyl)-4,4-difluorobut-3-enoate (2k): $27 \mathrm{mg}$, yield $52 \%$, chromatography on silica gel $[V($ petroleum ether $): V($ EtOAc $)=98: 2]$, colorless oil. ${ }^{1} \mathrm{H}$ NMR $(400 \mathrm{MHz}$, Chloroform- $d$ ) $\delta: 7.33(\mathrm{~d}, J=8.6 \mathrm{~Hz}$, $2 \mathrm{H}), 7.27(\mathrm{~d}, J=8.8 \mathrm{~Hz}, 2 \mathrm{H}), 4.12(\mathrm{q}, J=7.1 \mathrm{~Hz}, 2 \mathrm{H}), 3.37$ (s, 2H), $1.21(\mathrm{t}, J=7.1 \mathrm{~Hz}, 3 \mathrm{H}) ;{ }^{13} \mathrm{C}$ NMR $(101 \mathrm{MHz}$, $\left.\mathrm{CDCl}_{3}\right) \delta: 169.99 \sim 169.86(\mathrm{~m}), 154.79(\mathrm{dd}, J=292.7$, $289.9 \mathrm{~Hz}), 133.45,131.48(\mathrm{t}, J=4.0 \mathrm{~Hz}), 130.25,129.19$ $(\mathrm{t}, J=3.7 \mathrm{~Hz}), 128.94,128.73,86.49$ (dd, $J=22.1,17.6$ $\mathrm{Hz}), 61.22,33.71(\mathrm{~d}, J=2.3 \mathrm{~Hz}), 14.06 ;{ }^{19} \mathrm{~F}$ NMR $(376$ $\mathrm{MHz}$, Chloroform- $d$ ) $\delta$ : $-87.20(\mathrm{~d}, J=33.8 \mathrm{~Hz}),-88.36$ (d, $J=33.8 \mathrm{~Hz}$ ); HRMS (ESI) calcd for $\mathrm{C}_{12} \mathrm{H}_{11} \mathrm{ClF}_{2} \mathrm{O}_{2} \mathrm{Na}$ $[\mathrm{M}+\mathrm{Na}]^{+}$283.0314, found 283.0306.

Ethyl 3-(3-chlorophenyl)-4,4-difluorobut-3-enoate (21): $21 \mathrm{mg}$, yield $41 \%$, chromatography on silica gel $[V($ petroleum ether $): V($ EtOAc $)=98: 2]$, colorless oil. ${ }^{1} \mathrm{H}$ NMR $(400 \mathrm{MHz}$, Chloroform- $d$ ) $\delta: 7.26(\mathrm{~s}, 1 \mathrm{H}), 7.23 \sim$ $7.12(\mathrm{~m}, 3 \mathrm{H}), 4.06(\mathrm{q}, J=7.0 \mathrm{~Hz}, 2 \mathrm{H}), 3.30(\mathrm{~s}, 2 \mathrm{H}), 1.14(\mathrm{t}$, $J=7.1 \mathrm{~Hz}, 3 \mathrm{H}) ;{ }^{13} \mathrm{C}$ NMR (101 MHz, Chloroform- $d$ ) $\delta$ : 169.79 (dd, $J=4.2,2.8 \mathrm{~Hz}$ ), 154.97 (dd, $J=293.4,290.1$ $\mathrm{Hz}), 134.90(\mathrm{t}, J=4.0 \mathrm{~Hz}), 134.43,129.72,128.05(\mathrm{t}, J=$ $3.8 \mathrm{~Hz}), 127.74,126.07(\mathrm{t}, J=3.5 \mathrm{~Hz}), 86.55(\mathrm{dd}, J=22.4$, $17.5 \mathrm{~Hz}), 61.23,33.68$ (d, $J=2.2 \mathrm{~Hz}), 14.05 ;{ }^{19} \mathrm{~F}$ NMR (376 MHz, Chloroform- $d$ ) $\delta:-86.74(\mathrm{~d}, J=32.6 \mathrm{~Hz})$, -87.81 (d, $J=32.5 \mathrm{~Hz}$ ); HRMS (ESI) calcd for $\mathrm{C}_{12} \mathrm{H}_{11} \mathrm{ClF}_{2} \mathrm{O}_{2} \mathrm{Na}[\mathrm{M}+\mathrm{Na}]^{+}$283.0314, found 283.0310.

Ethyl 3-(4-bromophenyl)-4,4-difluorobut-3-enoate (2m): $40 \mathrm{mg}$, yield $66 \%$, chromatography on silica gel $[V$ (petroleum ether) $: V($ EtOAc $)=98: 2]$, colorless oil. ${ }^{1} \mathrm{H}$ NMR $(400 \mathrm{MHz}$, Chloroform- $d$ ) $\delta: 7.48(\mathrm{~d}, J=8.5 \mathrm{~Hz}$, $2 \mathrm{H}), 7.21(\mathrm{~d}, J=8.4 \mathrm{~Hz}, 2 \mathrm{H}), 4.12(\mathrm{q}, J=7.1 \mathrm{~Hz}, 2 \mathrm{H}), 3.37$ (s, $2 \mathrm{H}), 1.20(\mathrm{t}, J=7.1 \mathrm{~Hz}, 3 \mathrm{H}) ;{ }^{13} \mathrm{C}$ NMR $(101 \mathrm{MHz}$, Chloroform- $d$ ) $\delta$ : 169.87 (dd, $J=4.1,2.8 \mathrm{~Hz}), 154.74$ (dd, $J=292.8,289.9 \mathrm{~Hz}), 131.99$ (t, $J=4.0 \mathrm{~Hz}), 131.68,129.50$ $(\mathrm{t}, J=3.6 \mathrm{~Hz}), 121.58,86.57(\mathrm{dd}, J=22.2,17.6 \mathrm{~Hz})$, $61.20,33.65(\mathrm{~d}, J=2.2 \mathrm{~Hz}), 14.05 ;{ }^{19} \mathrm{~F}$ NMR $(376 \mathrm{MHz}$, Chloroform- $d$ ) $\delta:-87.21(\mathrm{~d}, J=33.7 \mathrm{~Hz}),-88.38(\mathrm{~d}$, $J=33.8 \mathrm{~Hz}$ ); HRMS (ESI) calcd for $\mathrm{C}_{12} \mathrm{H}_{11} \mathrm{BrF}_{2} \mathrm{O}_{2} \mathrm{Na}$ $[\mathrm{M}+\mathrm{Na}]^{+}$326.9808, found 326.9798 .

Ethyl 3-(3-bromophenyl)-4,4-difluorobut-3-enoate (2n): $41 \mathrm{mg}$, yield $67 \%$, chromatography on silica gel $[V($ petroleum ether $): V($ EtOAc $)=98: 2]$, colorless oil. ${ }^{1} \mathrm{H}$ 
NMR (400 MHz, Chloroform-d) $\delta: 7.49$ (s, 1H), 7.41 (d, $J=7.6 \mathrm{~Hz}, 1 \mathrm{H}), 7.30 \sim 7.18(\mathrm{~m}, 2 \mathrm{H}), 4.13(\mathrm{q}, J=7.1 \mathrm{~Hz}$, 2H), $3.36(\mathrm{~s}, 2 \mathrm{H}), 1.21(\mathrm{t}, J=7.1 \mathrm{~Hz}, 3 \mathrm{H}) ;{ }^{13} \mathrm{C}$ NMR $(101$ $\mathrm{MHz}$, Chloroform- $d$ ) $\delta$ : 169.77 (dd, $J=4.1,2.8 \mathrm{~Hz}$ ), $158.44 \sim 151.70(\mathrm{~m}), 135.18(\mathrm{t}, J=4.1 \mathrm{~Hz}), 130.94(\mathrm{t}, J=$ $3.7 \mathrm{~Hz}), 130.68,129.99,126.57(\mathrm{t}, J=3.5 \mathrm{~Hz}), 122.54$, 86.48 (dd, $J=22.5,17.6 \mathrm{~Hz}), 61.24,33.70$ (d, $J=2.4 \mathrm{~Hz}$ ), 14.06; ${ }^{19} \mathrm{~F}$ NMR (376 MHz, Methanol- $\left.d_{4}\right) \delta$ : -90.05 (d, $J=36.8 \mathrm{~Hz}),-91.16(\mathrm{~d}, J=36.3 \mathrm{~Hz})$; HRMS (ESI) calcd for $\mathrm{C}_{12} \mathrm{H}_{11} \mathrm{BrF}_{2} \mathrm{O}_{2} \mathrm{Na}[\mathrm{M}+\mathrm{Na}]^{+}$326.9808, found 326.9811 .

Ethyl 3-(benzofuran-3-yl)-4,4-difluorobut-3-enoate (2o): $28 \mathrm{mg}$, yield $78 \%$, chromatography on silica gel $[V($ petroleum ether $): V($ EtOAc $)=98: 2]$, colorless oil. ${ }^{1} \mathrm{H}$ NMR (400 MHz, Chloroform-d) $\delta: 7.67$ (s, 1H), 7.59 (d, $J=7.4 \mathrm{~Hz}, 1 \mathrm{H}), 7.50(\mathrm{~d}, J=8.0 \mathrm{~Hz}, 1 \mathrm{H}), 7.30$ (dt, $J=20.8$, $7.1 \mathrm{~Hz}, 2 \mathrm{H}), 4.14$ (q, J=7.1 Hz, 2H), 3.42 (s, 2H), 1.20 (t, $J=7.1 \mathrm{~Hz}, 3 \mathrm{H}) ;{ }^{13} \mathrm{C}$ NMR (101 MHz, Chloroform- $\left.d\right) \delta$ : 169.94 (dd, $J=4.1,2.9 \mathrm{~Hz}), 154.66$ (dd, $J=292.1,289.8$ $\mathrm{Hz}), 143.32$ (dd, $J=5.2,3.2 \mathrm{~Hz}), 124.68,122.93,120.49$ (d, $J=4.6 \mathrm{~Hz}), 113.69$ (dd, $J=4.2,3.2 \mathrm{~Hz}), 111.66,78.63$ (dd, $J=26.0,20.9 \mathrm{~Hz}), 61.21,33.88$ (d, $J=2.9 \mathrm{~Hz}), 14.03$; ${ }^{19} \mathrm{~F}$ NMR (376 MHz, Chloroform- $d$ ) $\delta$ : $-84.54(\mathrm{~d}, J=$ $32.1 \mathrm{~Hz}),-88.16(\mathrm{~d}, J=32.6 \mathrm{~Hz})$; HRMS (ESI) calcd for $\mathrm{C}_{14} \mathrm{H}_{13} \mathrm{~F}_{2} \mathrm{O}_{3}[\mathrm{M}+\mathrm{H}]^{+}$267.0833, found 267.0828.

Ethyl 4,4-difluoro-3-(phenanthren-9-yl) but-3-enoate (2p): $59 \mathrm{mg}$, yield $90 \%$, chromatography on silica gel $[V($ petroleum ether $): V($ EtOAc $)=98: 2]$, colorless solid. ${ }^{1} \mathrm{H}$ NMR (400 MHz, Chloroform- $d$ ) $\delta: 8.74(\mathrm{~d}, J=7.8 \mathrm{~Hz}$, $1 \mathrm{H}), 8.69(\mathrm{~d}, J=8.2 \mathrm{~Hz}, 1 \mathrm{H}), 7.98(\mathrm{~d}, J=7.9 \mathrm{~Hz}, 1 \mathrm{H}), 7.88$ $(\mathrm{d}, J=7.3 \mathrm{~Hz}, 1 \mathrm{H}), 7.77(\mathrm{~s}, 1 \mathrm{H}), 7.72 \sim 7.58(\mathrm{~m}, 4 \mathrm{H}), 4.08$ (q, $J=7.1 \mathrm{~Hz}, 2 \mathrm{H}), 3.48$ (s, 2H), 1.14 (t, $J=7.2 \mathrm{~Hz}, 3 \mathrm{H})$; ${ }^{13} \mathrm{C}$ NMR (101 MHz, Chloroform-d) $\delta: 170.01 \sim 169.74$ (m), 157.90 151.90 (m), 131.26, 130.75, 130.36, 130.10, $129.02(\mathrm{~d}, J=3.8 \mathrm{~Hz}), 128.77,127.15,126.87$ (d, $J=3.7$ $\mathrm{Hz}), 126.73,125.45,123.16,122.53,85.07$ (t, $J=22.4 \mathrm{~Hz})$, 61.07, $35.19(\mathrm{~d}, J=2.5 \mathrm{~Hz}), 14.02 ;{ }^{19} \mathrm{~F}$ NMR $(376 \mathrm{MHz}$, Chloroform- $d$ ) $\delta$ : $-86.44(\mathrm{~d}, J=34.8 \mathrm{~Hz}),-89.29$ (d, $J=34.8 \mathrm{~Hz}$ ); HRMS (ESI) calcd for $\mathrm{C}_{20} \mathrm{H}_{17} \mathrm{~F}_{2} \mathrm{O}_{2}[\mathrm{M}+\mathrm{H}]^{+}$ 327.1196, found 327.1188.

Ethyl 4,4-difluoro-3-(naphthalen-1-yl) but-3-enoate (2q): $50 \mathrm{mg}$, yield $90 \%$, chromatography on silica gel $[V$ (petroleum ether) $: V($ EtOAc $)=98: 2]$, colorless solid. ${ }^{1} \mathrm{H}$ NMR (400 MHz, Chloroform- $d$ ) $\delta: 7.94(\mathrm{~d}, J=8.1 \mathrm{~Hz}$, $1 \mathrm{H}), 7.91 \sim 7.82(\mathrm{~m}, 2 \mathrm{H}), 7.58 \sim 7.50(\mathrm{~m}, 2 \mathrm{H}), 7.48(\mathrm{~d}, J=$ $5.6 \mathrm{~Hz}, 2 \mathrm{H}), 4.08$ (q, $J=7.1 \mathrm{~Hz}, 2 \mathrm{H}), 3.48$ (s, 2H), 1.14 (d, $J=14.3 \mathrm{~Hz}, 3 \mathrm{H}) ;{ }^{13} \mathrm{C} \mathrm{NMR}(101 \mathrm{MHz}$, Chloroform- $d$ ) $\delta$ : $171.98 \sim 167.34(\mathrm{~m}), 154.52(\mathrm{dd}, J=291.4,288.2 \mathrm{~Hz})$, $133.77,131.35$ (d, $J=2.5 \mathrm{~Hz}), 130.45(\mathrm{dd}, J=4.4,1.8 \mathrm{~Hz})$, $128.72,127.70,128.54$, (dd, $J=3.4,1.5 \mathrm{~Hz}), 125.96$, $126.43,125.29,124.76,88.74 \sim 82.49(\mathrm{~m}), 61.00,35.37$ (d, $J=2.6 \mathrm{~Hz}), 13.96 ;{ }^{19} \mathrm{~F}$ NMR (376 MHz, Chloroform- $d$ ) $\delta$ : $-86.65(\mathrm{~d}, J=34.9 \mathrm{~Hz}),-89.59$ (d, $J=34.9 \mathrm{~Hz})$; HRMS (ESI) calcd for $\mathrm{C}_{16} \mathrm{H}_{14} \mathrm{~F}_{2} \mathrm{O}_{2} \mathrm{Na}[\mathrm{M}+\mathrm{Na}]^{+}$299.0860, found 299.0868.

Ethyl 4, 4-difluoro-3-(thiophen-3-yl) but-3-enoate (2r): $39 \mathrm{mg}$, yield $84 \%$, chromatography on silica gel
$[V($ petroleum ether $): V($ EtOAc $)=98: 2]$, colorless oil. ${ }^{1} \mathrm{H}$ NMR (400 MHz, Chloroform-d) $\delta$ : 7.32 (dd, $J=5.2,3.1$ $\mathrm{Hz}, 1 \mathrm{H}), 7.23$ (s, 1H), 7.18 (d, $J=4.9 \mathrm{~Hz}, 1 \mathrm{H}), 4.15$ (q, $J=$ $7.1 \mathrm{~Hz}, 2 \mathrm{H}), 3.38(\mathrm{~s}, 2 \mathrm{H}), 1.23(\mathrm{t}, J=7.1 \mathrm{~Hz}, 3 \mathrm{H}) .{ }^{13} \mathrm{C}$ NMR (101 MHz, Chloroform-d) $\delta: 170.33 \sim 170.06(\mathrm{~m})$, $155.04(\mathrm{dd}, J=294.1,288.4 \mathrm{~Hz}), 133.11$ (dd, $J=5.4,3.4$ $\mathrm{Hz}), 126.55$ (dd, $J=7.0,2.3 \mathrm{~Hz}), 125.76,121.91(\mathrm{t}, J=5.5$ $\mathrm{Hz}), 83.81$ (dd, $J=23.5,17.5 \mathrm{~Hz}), 61.17,33.40$ (d, $J=3.1$ $\mathrm{Hz}), 14.05$; ${ }^{19} \mathrm{~F}$ NMR (376 MHz, Chloroform- $\left.d\right) \delta$ : $-85.32(\mathrm{~d}, J=34.0 \mathrm{~Hz}),-89.32(\mathrm{~d}, J=34.0 \mathrm{~Hz})$; HRMS (ESI) calcd for $\mathrm{C}_{10} \mathrm{H}_{11} \mathrm{~F}_{2} \mathrm{O}_{2 \mathrm{~S}}[\mathrm{M}+\mathrm{H}]^{+}$233.0448, found 233.0449 .

Butyl 4,4-difluoro-3-phenylbut-3-enoate (2s): $44 \mathrm{mg}$, yield $87 \%$, chromatography on silica gel [ $V$ (petroleum ether) $: V($ EtOAc $)=98: 2]$, colorless oil. ${ }^{1} \mathrm{H}$ NMR $(400$ $\mathrm{MHz}$, Chloroform- $d$ ) $\delta: 7.35(\mathrm{~d}, J=5.5 \mathrm{~Hz}, 4 \mathrm{H}), 7.28(\mathrm{dt}$, $J=4.9,2.5 \mathrm{~Hz}, 1 \mathrm{H}), 4.07(\mathrm{t}, J=6.6 \mathrm{~Hz}, 2 \mathrm{H}), 3.40$ (t, $J=$ $2.1 \mathrm{~Hz}, 2 \mathrm{H}), 1.55$ (dt, $J=14.6,6.7 \mathrm{~Hz}, 2 \mathrm{H}), 1.29$ (dq, $J=$ 14.6, 7.4 Hz, 2H), 0.89 (t, $J=7.4 \mathrm{~Hz}, 3 \mathrm{H}) ;{ }^{13} \mathrm{C} \mathrm{NMR} \mathrm{(101}$ MHz, Chloroform- $d$ ) $\delta: 170.19$ (dd, $J=4.1,2.8 \mathrm{~Hz}$ ), 154.80 (dd, $J=292.4,289.0 \mathrm{~Hz}), 133.04$ (t, $J=3.8 \mathrm{~Hz})$, $128.48,127.90 \sim 127.71(\mathrm{~m}), 127.51,87.23(\mathrm{dd}, J=21.5$, $17.7 \mathrm{~Hz}), 64.97,33.91,33.89$ (d, $J=2.6 \mathrm{~Hz}), 30.51,18.97$, 13.58; ${ }^{19} \mathrm{~F}$ NMR (376 MHz, Methanol- $\left.d_{4}\right) \delta$ : -91.09 (d, $J=40.1 \mathrm{~Hz}),-92.25$ (d, $J=39.5 \mathrm{~Hz})$; HRMS (ESI) calcd for $\mathrm{C}_{14} \mathrm{H}_{17} \mathrm{~F}_{2} \mathrm{O}_{2}[\mathrm{M}+\mathrm{H}]^{+}$255.1196, found 255.1195.

Tert-butyl 4,4-difluoro-3-phenylbut-3-enoate (2t): 26 $\mathrm{mg}$, yield $52 \%$, chromatography on silica gel [ $V$ (petroleum ether $): V($ EtOAc $)=98: 2]$, colorless oil. ${ }^{1} \mathrm{H}$ NMR $(400$ $\mathrm{MHz}$, Chloroform- $d$ ) $\delta: 7.38 \sim 7.28(\mathrm{~m}, 4 \mathrm{H}), 7.34 \sim 7.25$ $(\mathrm{m}, 1 \mathrm{H}), 3.34(\mathrm{t}, J=2.3 \mathrm{~Hz}, 2 \mathrm{H}), 1.39(\mathrm{~s}, 9 \mathrm{H}) ;{ }^{13} \mathrm{C} \mathrm{NMR}$ (101 MHz, Chloroform-d) $\delta$ : 169.31 (dd, $J=4.0,2.8 \mathrm{~Hz}$ ), $154.71(\mathrm{dd}, J=292.1,288.7 \mathrm{~Hz}), 133.26(\mathrm{t}, J=3.9 \mathrm{~Hz})$, $128.40,127.87(\mathrm{t}, J=3.5 \mathrm{~Hz}), 127.40,87.63(\mathrm{dd}, J=21.6$, $17.3 \mathrm{~Hz}), 81.27,35.09(\mathrm{~d}, J=2.5 \mathrm{~Hz}), 27.85 ;{ }^{19} \mathrm{~F}$ NMR (376 MHz, Methanol- $\left.d_{4}\right) \delta$ : -91.07 (d, $J=40.2 \mathrm{~Hz}$ ), - $92.41(\mathrm{~d}, J=39.6 \mathrm{~Hz})$; HRMS (ESI) calcd for $\mathrm{C}_{14} \mathrm{H}_{17} \mathrm{~F}_{2} \mathrm{O}_{2}[\mathrm{M}+\mathrm{H}]^{+}$255.1196, found 255.1198.

Allyl 4,4-difluoro-3-phenylbut-3-enoate (2u): $32 \mathrm{mg}$, yield $68 \%$, chromatography on silica gel [ $V$ (petroleum ether $): V($ EtOAc $)=98: 2]$, colorless oil. ${ }^{1} \mathrm{H}$ NMR $(400$ $\mathrm{MHz}$, Chloroform-d) $\delta: 7.35$ (d, $J=5.7 \mathrm{~Hz}, 4 \mathrm{H}), 7.30 \sim$ 7.27 (m, 1H), 5.85 (ddt, $J=16.6,11.0,5.7 \mathrm{~Hz}, 1 \mathrm{H}), 5.29 \sim$ $5.13(\mathrm{~m}, 2 \mathrm{H}), 4.57(\mathrm{~d}, J=5.5 \mathrm{~Hz}, 2 \mathrm{H}), 3.44(\mathrm{~s}, 2 \mathrm{H}) ;{ }^{13} \mathrm{C}$ NMR (101 MHz, Chloroform- $d) \delta: 169.93 \sim 169.66(\mathrm{~m})$, 154.86 (dd, $J=292.5,289.3 \mathrm{~Hz}), 132.95$ (t, $J=3.8 \mathrm{~Hz}$ ), $131.73,128.52,127.86(\mathrm{t}, J=3.5 \mathrm{~Hz}), 127.59,118.37$, 87.08 (dd, $J=21.4,18.0 \mathrm{~Hz}), 65.68,33.83$ (d, $J=2.6 \mathrm{~Hz}$ ); ${ }^{19} \mathrm{~F}$ NMR (376 MHz, Chloroform- $d$ ) $\delta$ : -86.44 (d, $J=$ $34.8 \mathrm{~Hz}$ ), -89.29 (d, $J=34.7 \mathrm{~Hz}$ ); HRMS (ESI) calcd for $\mathrm{C}_{13} \mathrm{H}_{13} \mathrm{~F}_{2} \mathrm{O}_{2}[\mathrm{M}+\mathrm{H}]^{+}$239.0883, found 239.0880.

2,2,2-Trifluoroethyl 4,4-difluoro-3-phenylbut-3-enoate (2v): $23 \mathrm{mg}$, yield $42 \%$, chromatography on silica gel $[V($ petroleum ether $): V($ EtOAc $)=98: 2]$, colorless oil. ${ }^{1} \mathrm{H}$ NMR (400 MHz, Chloroform- $d) \delta: 7.41 \sim 7.27(\mathrm{~m}, 5 \mathrm{H})$, 4.45 (q, $J=8.4 \mathrm{~Hz}, 2 \mathrm{H}), 3.52(\mathrm{t}, J=2.1 \mathrm{~Hz}, 2 \mathrm{H}) ;{ }^{13} \mathrm{C} \mathrm{NMR}$ (101 MHz, Chloroform- $d$ ) $\delta: 168.65$ (dd, $J=4.3,2.9 \mathrm{~Hz}$ ), 
$158.17 \sim 151.73(\mathrm{~m}), 132.56 \sim 132.28(\mathrm{~m}), \quad 128.65$, $128.10 \sim 127.55(\mathrm{~m}), 122.70(\mathrm{q}, J=277.1 \mathrm{~Hz}), 86.48(\mathrm{dd}$, $J=21.2,18.9 \mathrm{~Hz}), 60.76(\mathrm{q}, J=36.9 \mathrm{~Hz}), 33.35$ (d, $J=2.6$ $\mathrm{Hz}) ;{ }^{19} \mathrm{~F}$ NMR (376 MHz, Chloroform- $d$ ) $\delta$ : -70.30 , -87.50 (d, $J=34.5 \mathrm{~Hz}),-88.58$ (d, $J=34.6 \mathrm{~Hz}$ ); HRMS (ESI) calcd for $\mathrm{C}_{12} \mathrm{H}_{10} \mathrm{~F}_{5} \mathrm{O}_{2}[\mathrm{M}+\mathrm{H}]^{+}$281.0601, found 281.0606 .

$\mathrm{N}$-Benzyl-4,4-difluoro-3-phenylbut-3-enamide (2w): 46 mg, yield $81 \%$, chromatography on silica gel $[V$ (petroleum ether) $: V($ EtOAc $)=98: 2]$, colorless oil. ${ }^{1} \mathrm{H}$ NMR $(400$ MHz, Chloroform- $d$ ) $\delta$ : $7.28(\mathrm{~d}, J=4.4 \mathrm{~Hz}, 4 \mathrm{H}), 7.27 \sim$ $7.20(\mathrm{~m}, 1 \mathrm{H}), 7.21 \sim 7.13(\mathrm{~m}, 3 \mathrm{H}), 7.01 \sim 6.93(\mathrm{~m}, 2 \mathrm{H})$, $5.83(\mathrm{~s}, 1 \mathrm{H}), 4.31(\mathrm{~d}, J=5.7 \mathrm{~Hz}, 2 \mathrm{H}), 3.31(\mathrm{t}, J=2.2 \mathrm{~Hz}$, $2 \mathrm{H}) ;{ }^{13} \mathrm{C}$ NMR (101 MHz, Chloroform-d) $\delta: 168.87 \sim$ $168.61(\mathrm{~m}), 154.79$ (dd, $J=294.5,289.4 \mathrm{~Hz}), 132.44$ (t, $J=3.8 \mathrm{~Hz}), 128.74,128.60,127.88 \sim 127.66(\mathrm{~m}), 127.41$, $126.18(\mathrm{~d}, J=3.2 \mathrm{~Hz}), 87.71(\mathrm{dd}, J=21.1,15.9 \mathrm{~Hz}), 43.62$, 35.70 (d, $J=1.9 \mathrm{~Hz}) ;{ }^{19} \mathrm{~F}$ NMR (376 MHz, Chloroform- $d$ ) $\delta$ : $-86.70(\mathrm{~d}, J=33.5 \mathrm{~Hz}),-87.41(\mathrm{~d}, J=33.8 \mathrm{~Hz})$; HRMS (ESI) calcd for $\mathrm{C}_{17} \mathrm{H}_{16} \mathrm{~F}_{2} \mathrm{NO}[\mathrm{M}+\mathrm{H}]^{+}$288.1200, found 288.1192 .

Supporting Information The preparation and characterization of substrate $1 \mathbf{a} \sim \mathbf{1 w} .{ }^{1} \mathrm{H} \mathrm{NMR}$ and $1{ }^{3} \mathrm{C}$ NMR spectra for all pure products $\mathbf{2 a} \sim \mathbf{2} \mathbf{w}$, the $\mathrm{CV}$ and $\mathrm{SWV}$ experiments. The Supporting Information is available free of charge via the Internet at http://sioc-journal.cn/.

\section{References}

[1] (a) McDonald, I. A.; Lacoste, J. M.; Bey, P.; Palfreyman, M. G.; Zreika, M. J. Med. Chem. 1985, 28, 186. (b) Sayre, L. M. WO2007005737A2. 2007

[2] Okada, H.; Morita, M.; Ueda, T.; Takeo, H.; Kominami, H.; Kiriyama, K.; Nakamoto, K.; Yoshida, Y. WO2004052872A1, 2004

[3] Koley, S.; Altman, R. A. Isr. J. Chem. 2020, 60, 313.

[4] (a) Gao, B.; Zhao, Y.; Ni, C.; Hu, J. Org. Lett. 2014, 16, 102. (b) Gao, B.; Zhao, Y.; Hu, J. Angew. Chem., Int. Ed. 2015, 54, 638.

[5] (a) Tian, P.; Feng, C.; Loh, T.-P. Nat. Commun. 2015, 6, 7472. (b) Tian, P.; Wang, C.-Q.; Cai, S.-H.; Song, S.; Ye, L.; Feng, C.; Loh, T.-P. J. Am. Chem. Soc. 2016, 138, 15869.

(c) Cai, S.-H.; Ye, L.; Wang, D.-X.; Wang, Y.-Q.; Lai, L.-J.; Zhu, C.; Feng, C.; Loh, T.-P. Chem. Commun. 2017, 53, 8731.

(d) Tang, H.-J.; Lin, L.-Z.; Feng, C.; Loh, T.-P. Angew. Chem., Int. Ed. 2017, 56, 9872.

(e) Zhu, C.; Song, S.; Zhou, L.; Wang, D.-X.; Feng, C.; Loh, T.-P. Chem. Commun. 2017, 53, 9482.

(f) Tang, H.-J.; Zhang, Y.-F.; Jiang, Y.-W.; Feng, C. Org. Lett. 2018, 20, 5190 .

(g) Zhou, L.; Zhu, C.; Loh, T.-P.; Feng, C. Chem. Commun. 2018, $54,5618$.

(h) Liu, H.; Ge, L.; Wang, D.-X.; Chen, N.; Feng, C. Angew. Chem., Int. Ed. 2019, 58, 3918.

(i) Zhou, L.; Zhu, C.; Bi, P.; Feng, C. Chem. Sci. 2019, 10, 1144.

(j) Zhu, C.; Zhang, Y.-F.; Liu, Z.-Y.; Zhou, L.; Liu, H.; Feng, C. Chem. Sci. 2019, 10, 6721.

(k) Cao, Z.-C.; Liu, J.-C.; Chu, Y.-Q.; Zhao, F.-M.; Zhu, Y.-H.; She, Y.-B. Chin. J. Org. Chem. 2019, 39, 2499 (in Chinese). (曹志成, 刘建超, 褚有群, 赵峰鸣, 朱英红, 余远斌, 有机化学
2019, 39, 2499.)

(1) Du, H.-W.; Sun, J.; Gao, Q.-S.; Wang, J.-Y.; Wang, H.; Xu, Z.; Zhou, M.-D. Org. Lett. 2020, 22, 1542.

[6] Zubkov, M. O.; Kosobokov, M. D.; Levin, V. V.; Kokorekin, V. A.; Korlyukov, A. A.; Hu, J.; Dilman, A. D. Chem. Sci. 2020, 11, 737.

[7] Liu, C.; Zhu, C.; Cai, Y.; Yang, Z.; Zeng, H.; Chen, F.; Jiang, H. Chem.-Eur. J. 2020, 26, 1953.

[8] Chelucci, G. Chem. Rev. 2012, 112, 1344.

[9] Nihei, T.; Iwai, N.; Matsuda, T.; Kitazume, T. J. Org. Chem. 2005, $70,5912$.

[10] Cao, C.-R.; Ou, S.; Jiang, M.; Liu, J.-T. Tetrahedron Lett. 2017, 58 , 482.

[11] Wang, S.; Cheng, B.-Y.; Sršen, M.; König, B. J. Am. Chem. Soc. 2020, $142,7524$.

[12] Yu, J.; Lin, J.-H.; Xiao, J.-C. Chin. J. Org. Chem. 2019, 39, 265 (in Chinese).

(于蛟, 林锦鸿, 肖吉昌, 有机化学, 2019, 39, 265.)

[13] Guo, S.; Yang, P.; Zhou, J. Chem. Commun. 2015, 51, 12115.

[14] (a) Chen, J.; Lv, S.; Tian, S. ChemSusChem 2019, 12, 115.

(b) Gandeepan, P.; Kaplaneris, N.; Santoro, S.; Vaccaro, L.; Ackermann, L. ACS Sustainable Chem. Eng. 2019, 7, 8023.

(c) Mei, H.; Yin, Z.; Liu, J.; Sun, H.; Han, J. Chin. J. Chem. 2019, 37,292

(d) Meyer, T. H.; Finger, L. H.; Gandeepan, P.; Ackermann, L. Trends Chem. 2019, 1, 63.

(e) Qiu, Y.; Struwe, J.; Ackermann, L. Synlett 2019, 30, 1164

(f) Song, C.; Liu, K.; Dong, X.; Chiang, C.-W.; Lei, A. Synlett 2019, 30, 1149.

(g) Wang, H.; Gao, X.; Lv, Z.; Abdelilah, T.; Lei, A. Chem. Rev. 2019, 119, 6769 .

(h) Xiong, P.; Xu, H.-C. Acc. Chem. Res. 2019, 52, 3339.

(i) Ye, Z.; Zhang, F. Chin. J. Chem. 2019, 37, 513.

(j) Yuan, Y.; Lei, A. Acc. Chem. Res. 2019, 52, 3309.

(k) Zhang, H.-Y.; Tang, R.-P.; Shi, X.-L.; Jie, L.; Wu, J.-W. Chin. J. Org. Chem. 2019, 39, 1837 (in Chinese).

(张怀远, 唐蓉萍, 石星丽, 颕林, 伍家卫, 有机化学, 2019, 39, 1837.)

(1) Ackermann, L. Acc. Chem. Res. 2020, 53, 84.

(m) Jiao, K.-J.; Xing, Y.-K.; Yang, Q.-L.; Qiu, H.; Mei, T.-S. Acc. Chem. Res. 2020, 53, 300.

(n) Li, M.; Hong, J.; Xiao, W.; Yang, Y.; Qiu, D.; Mo, F. ChemSusChem 2020, 13, 1661.

(o) Rockl, J. L.; Pollok, D.; Franke, R.; Waldvogel, S. R. Acc. Chem. Res. 2020, 53, 45 .

(p) Wang, P.; Gao, X. L.; Huang, P. F.; Lei, A. W. ChemCatChem 2020, 12,27

(q) Wang, X.-Y.; Xu, X.-T.; Wang, Z.-H.; Fang, P.; Mei. T.-S. Chin J. Org. Chem. 2020, 40, 3738 (in Chinese).

(王向阳, 徐学涛, 王振华, 方萍, 梅天胜, 有机化学, 2020, 40, 3738.)

[15] Peters, D. G.; McGuire, C. M.; Pasciak, E. M.; Peverly, A. A.; Strawsine, L. M.; Wagoner, E. R.; Barnes, J. T. Rev. Soc. Quim. Mex. 2017, 58, 287.

[16] Huang, H.; Lambert, T. H. Angew. Chem., Int. Ed. 2020, 59, 658.

[17] (a) Uneyama, K.; Kato, T. Tetrahedron Lett. 1998, 39, 587.

(b) Uneyama, K.; Maeda, K.; Kato, T.; Katagiri, T. Tetrahedron Lett. 1998, 39, 3741 .

(c) Uneyama, K.; Mizutani, G. Chem. Commun. 1999, 613.

(d) Uneyama, K.; Mizutani, G.; Maeda, K.; Kato, T. J. Org. Chem. 1999, 64, 6717.

[18] (a) Liu, X.; Liu, R.; Qiu, J.; Cheng, X.; Li, G. Angew. Chem., Int. Ed., 2020, 59, 13962.

(b) Li, J.; He, L.; Liu, X.; Cheng, X.; Li, G. Angew. Chem., Int. Ed. 2019, $58,1759$. 